\title{
Recovering Image Structure by Model-Based Interaction Map
}

\author{
Georgy Gimel'farb \\ CITR, Department of Computer Science, Tamaki Campus, The University of \\ Auckland, Private Bag 92019, Auckland, New Zealand
}

\begin{abstract}
We address modelling of stochastic image textures by Gibbs random fields with a translation invariant structure of multiple pairwise pixel interactions. The characteristic interaction structure and strengths (Gibbs potentials) are learnt from a given training sample by analytic and stochastic approximation of the unconditional or conditional maximum likelihood estimates of the potentials. The interaction structure is revealed by a model-based interaction map showing the relative contributions of each interaction to a total Gibbs energy. Features of the inter:uction maps are discussed and illustrated by experiments with various natural textures.
\end{abstract}

\section{Introduction: Structure and Texture}

Notions of image texture and spatial image structure are widely used in today's image processing and recognition although both terms lack formal and precise definitions [10]. The texture model involves basic grey level (or color, or multiband) texture primitives which texture elements, called textons [11] or texels [10], are built from. Spatial interactions between the texels govern their spatial arrangement and particular signal values in each the primitive. In this context, the interaction has no direct physical meaning and deals only with relative frequencies of particular spatial signal combinations formed by texels: the more frequent the combination, the stronger the interaction. Such interpretation suggests that the basic property of an image to be a homogeneous texture is a spatial selfsimilarity. It means a (statistical) repetitiveness of specific signal combinations over the image. The definition is still informal but, at least, proposes a way of building the formal one which has to specify quantitatively what and how has to repeat in a texture.

For a human, the spatial self-similarity, or repetitiveness, is quite definitive. But, it is very hard (if possible) to give it a general constructive formal definition. An early self-similarity concept [4] referred to an image patch of minimum size that can be considered as the texture and to a binary similarity relation between the patches. But, no ways were proposed how to find them in practice. More elaborated approach in [12] involves distances between conditional probability distributions of a particular function of the grey level configurations in the patches as a quantitative measure of the similarity. The patches are certain translation-invariant connected pixel subsets which are equivalent under a given 
group of transformations. But, generally it is unclear how to search for these patches and functions for testing the spatial self-similarity of a given image.

To avoid the impracticable generalisations, the self-similarity concept is restricted here to pixel pairs as the (simplest) texels and to repetitive conditional probability distributions of signals in the texels of the same type. Such a restriction allows for a precise definition of the spatial self-similarity in terms of quantitative parameters of particular Markov or non-Markov Gibbs random fields (GRF) with multiple pairwise pixel interactions proposed in $[6,7]$. Images are considered as samples of a GRF defined by a Gibbs probability distribution (GPD). Parameters of the GPD govern a geometric structure and quantitative strengths of pixel interactions.

The structure is represented by a neighbourhood graph joining in the lattice supporting the images all the interacting pixel pairs called neighbours [1]. For a translation invariant structure, the graph contains several families of the pixel pairs. The pairs of a single family have the same relative arrangement of the pixels and differ only by their absolute positions in the lattice. The inter-pixel shifts specifying the families can be arbitrary and depend on a texture type to be modelled.

The GPD is factored over these families, each factor being an exponential function of a (Gibbs) potential. Each family has its own potential that depends on the signals in the pixel pair. The potential defines a quantitative interaction strength in this pair. In the Markov/Gibbs models these pairs are cliques, or complete subgraphs of the neighbourhood graph [1]. In the simple non-Markov Gibbs models introduced in [7] these pairs are not the cliques because the neigbourhood of each pixel coincides, in principle, with the total lattice. But, they represent still most significant part of the "lattice-wide" interactions. For brevity, we refer below to them by the same terms "cliques" and "clique families".

Basically, such a model leaves aside most of the textures because usually they have larger and more diverse texels and greater geometric differences between the self-similar parts. Nevertheless, the experiments show that a sufficient number of natural and artificial image textures are modelled quite adequately. Regarding the homogeneous textures, the models with multiple pairwise pixel interactions overcome some drawbacks of more traditional automodels considered, for instance, in [1,3] in two directions: (i) they allow to learn both the characteristic interaction structure and the Gibbs potentials from a given training sample, and (ii) some simple gray range transformations (such as arbitrary shifts or linear stretches of the image grey ranges) that have no effect on the texture type can be taken into account by choosing a particular form of the potentials for the Markov/Gibbs models [6] or by involving specific non-Markov Gibbs image models [7].

The paper is organised as follows. Section 2 presents a particular Gibbs image model with multiple pairwise pixel interactions and the model-based interaction map that allows to reveal the structure of most characteristic interactions. Section 3 presents experimental results for various natural image textures and gives some conclusions. 


\section{Model-Based Interaction Maps}

The typical Gibbs image model with multiple pairwise pixel interactions is as follows (see $[6,7]$ for more details):

$$
\operatorname{Pr}(\mathbf{g} \mid \mathbf{V})=\frac{1}{Z_{\mathbf{V}}} \cdot \exp \left(\sum_{a \in \mathbf{A}} e_{a}(\mathbf{g} \mid \mathbf{V})\right) .
$$

where $\mathbf{g}$ is a greyscale image and $\mathbf{V}=\left(\mathbf{V}_{a}: a \in \mathbf{A}\right)$ is the potential vector containing the partial vectors $\mathbf{V}_{a}$ defined for the grey level differences in the cliques of each family $a \in \mathbf{A}$. Here, $e_{a}(\mathbf{g} \mid \mathbf{V})$ is a partial (Gibbs) energy for the family $a$ and $Z_{\mathbf{V}}$ is a normalising factor. The partial Gibbs energies can be represented by dot products of the potential vectors and the vectors of the gray level difference histograms (GLDH) collected over the image $[6,7]$ :

$$
\forall a \in \mathbf{A} \quad e_{a}\left(\mathbf{g} \mid \mathbf{V}_{a}\right)=\mathbf{V}_{a} \bullet \mathbf{H}_{a}(\mathbf{g})
$$

so that the total Gibbs energy of the interactions in Eq. (1) is as follows:

$$
E(\mathbf{g} \mid \mathbf{V})=\sum_{a \in \mathbf{A}} e_{a}(\mathbf{g} \mid \mathbf{V})=\mathbf{V} \bullet \mathbf{H}(\mathbf{g}) .
$$

The model parameters $(\mathbf{A}, \mathbf{V})$ can be learnt from a given training sample $\mathbf{g}^{\circ}$ using either the unconditional or the conditional maximum likelihood estimate (MLE) of the potentials $[6,7,8]$. The learning starts from an analytic first approximation of the potentials. This approximation, computed from the GLDHs for the model in Eq. (1), enables to compare relative strengths of a great many possible pairwise interactions and to recover most characteristic ones representing a given texture type. Then, the desired potential estimates for the chosen families are refined by a stochastic approximation technique.

Let $\mathbf{W}$ be a given search set of a big many neighbours covering a given large range of possible inter-pixel shifts: $\mathbf{W}=\left\{(\mu, \nu):|\mu| \leq \mu_{\max } ;|\nu| \leq \nu_{\max }\right\}$. A rich variety of the clique families, defined by the search set $\mathbf{W}$, are compared by their relative Gibbs energies $e_{(\mu, \nu),[0]}\left(\mathbf{g}^{\circ}\right)$ computed for a given training sample $\mathbf{g}^{\circ}$ by using first analytic approximations of the potentials. In so doing, the energy values over the search set $\mathbf{W}$ are represented as a 2D "energy function graph" $e\left(\mu, \nu \mid \mathbf{g}^{\circ}\right)$ with the planar cartesian co-ordinates $(\mu, \nu)$. This representation constitutes a model-based interaction map which shows relative contributions of each clique family to the total energy of Eq. (3) and can be displayed, for a visual analysis, in a greyscale or color form $[6,9]$.

A simple technique for finding most characteristic clique families that comprise the desired interaction structure of the model in Eq. (1) is based on a direct thresholding of the map:

$$
\mathbf{A}=\left\{a=(\mu, \nu):(\mu, \nu) \in \mathbf{W} ; e_{\mu, \nu}\left(\mathbf{g}^{\circ}\right)>\theta\right\}
$$

where $\theta$ denotes a given threshold. In our experiments $[6,9]$ it has been chosen as a function either of the mean relative energy $\bar{e}$ and standard deviation $\sigma_{e}$ in 
the interaction map: $\theta=\bar{e}+c \cdot \sigma_{e}$, where $c=3 \ldots 4$, or of the maximum relative energy: $\theta=c \cdot e_{\max }$ where $c=0.25 \ldots 0.35$. But, further theoretical investigation is needed for optimising such a search.

\section{Experimental Results and Conclusions}

\subsection{Interaction maps for natural and simulated textures}

Figures 1 and 2 display the natural texture samples from [2], simulated textures, and their interaction maps. Figure 1 shows, respectively, four digitized fragments $128 \times 128$ of the weakly homogeneous natural texture D3 (Reptile Skin) and the corresponding interaction maps representing 3280 clique families of the search set $\mathbf{W}$ formed with the parameters $\mu_{\max }=40 ; \nu_{\max }=40$. The maps shown contain two square boxes $(2 \times 2$ pixels in Figures 1 and 2$)$ with relative coordinates $(\mu, \nu)$ and $(-\mu,-\nu)$ per family with the intra-clique shift $(\mu, \nu) \in \mathbf{W}$. The origin of Cartesian coordinates $(\mu, \nu)$ is marked by a white square. For visual representation, the energies $e\left(\mu, \nu \mid \mathbf{g}^{\circ}\right)$ are coded by the grey levels: the darker the box, the greater the energy and the stronger the interaction. Figure 2 shows the like fragments and interaction maps for the natural texture D14 (Woven Aluminum Wire). It is obvious that the presented textures possess the selfsimilarity in the introduced "model-based" sense if the texels are sufficiently large to represent the repetitive parts of these patterns. But, as regarding the translation invariant pairwise interactions, this self-similarity is rather weak, especially for the texture D3. Nonetheless, the simulation experiments, discussed below, show that the model of Eq. (1) reflects most characteristic features which are perceived visually in these textures.

The shown interaction maps exhibit relative contributions of different clique families presented in the search set $\mathbf{W}$ to the imaginary Gibbs model that contains all the families. This allows for approximating a given texture type by a reduced model with zero-valued potentials for the families with too weak interaction strengths. Generally, this suggests that all the models have the same interaction structure that correspond to the largest possible search set $\mathbf{W}$ and differ only by the potentials: the non-zero values for the characteristic clique families and zero values for all the other families. It is this feature that simplifies comparisons of different textures, say, for the image retrieval [9].

Here, a heuristic search for most characteristic clique families is done by thresholding the interaction map with the threshold $\theta=\bar{e}+c \cdot \sigma_{e}$. The interaction structures in Figures 1 and 2, learnt with $c=3$, have similar geometric forms for different samples which reflect basic visually perceived hexagonal or tetragonal patterns of these textures. The structures of most homogeneous samples include approximately the same numbers of the clique families: say, $58 \ldots 60$ in Figure 1 (the samples T-D3a, T-D3c, T-D3d) and $71 \ldots 76$ in Figure 2 (the samples TD14a - T-D14c). Local inhomogeneities affect the learnt structures. For instance, due to notable cell size changes and to a non-homogeneous cell arrangement for the sample T-D3b in Figure 1 some characteristic long-range interactions are missing from the structure ST3b. 


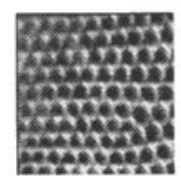

T-D3a

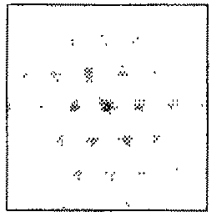

MT3a

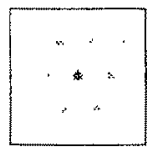

ST3a

(60 families)

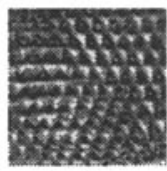

G-D3a

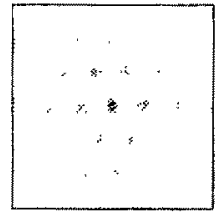

MG3a

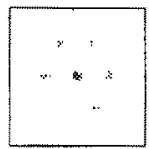

SG3a

(57 families)

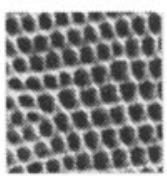

T-D3b

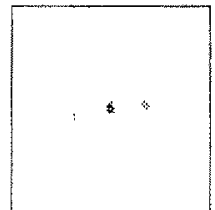

MT3b

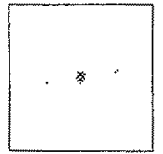

ST3b

(50 families)

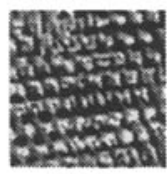

G-D3b

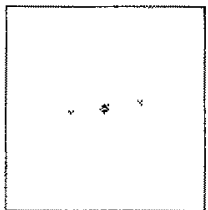

MG3b

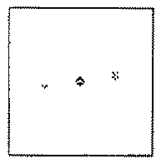

SG3b

(45 families)

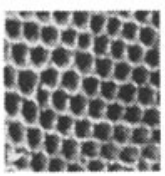

T-D3c

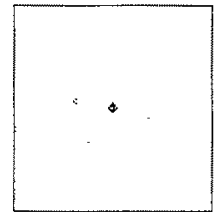

MT3c

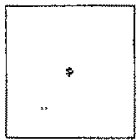

ST3c

(58 families)

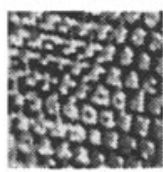

G-D3c

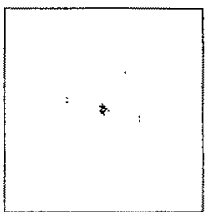

MG3c

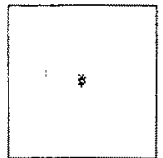

SG3c

(59 families)

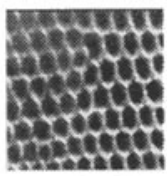

T-D3d

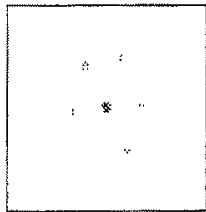

MT3d

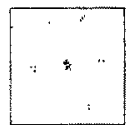

ST3d

(60 families)

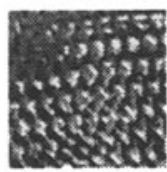

G-D3d

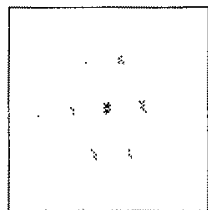

MG3d

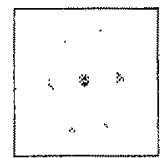

SG3d

(60 families)

Fig. 1. Texture type D3 (Reptile skin): training (T) and generated (G) samples, their interaction maps (MT, MG), and recovered structures (ST, SG) with the learnt number of the clique families. 


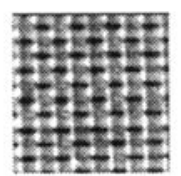

T-D14a

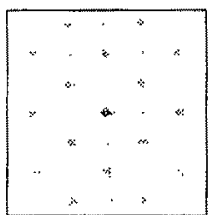

MT14a

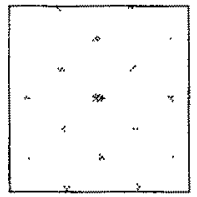

ST14a

(71 families)

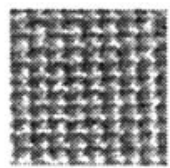

G-D14a

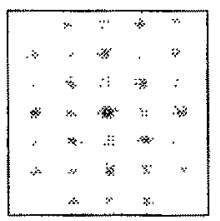

MG14a

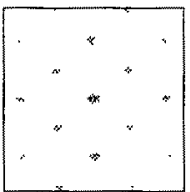

SG14a

(64 families)

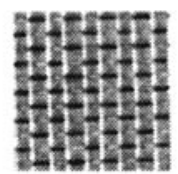

T-D14b

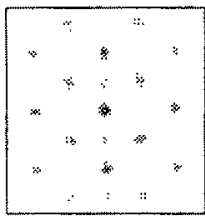

MT14b

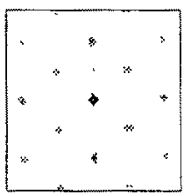

ST14b

(76 families)

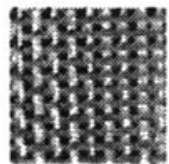

G-D14b

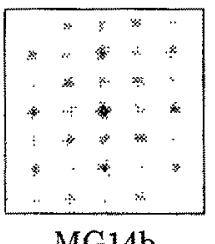

MG14b

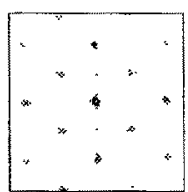

SG14b

(74 families)

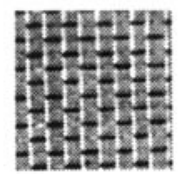

T-D14c

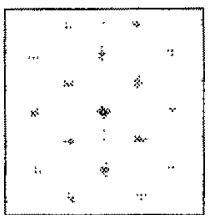

MT14c

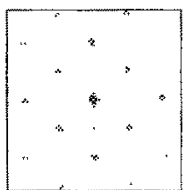

ST14c

(73 families)

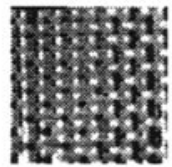

G-D14c

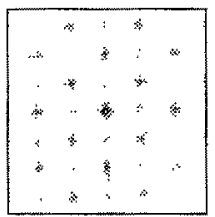

MG14c

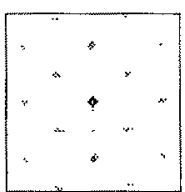

SG14c

(73 families)

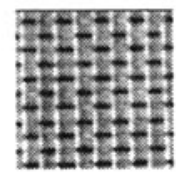

T-D14d

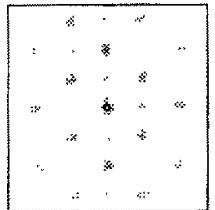

MT14d

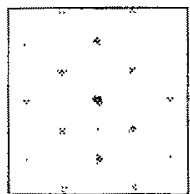

ST14d

(79 families)

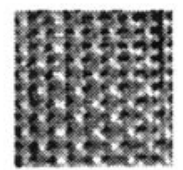

G-D14d

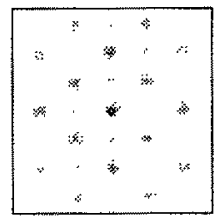

MG14d

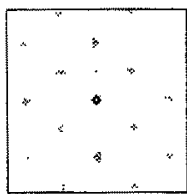

SG14d

(79 families)

Fig. 2. : Texture type D14 (Woven aluminum wire): training (T) and generated $(\mathrm{G})$ samples, their interaction maps (MT, MG), and recovered structures (ST, SG) with the learnt number of the clique families. 


\subsection{Comparing natural and simulated textures}

Figures 1 and 2 demonstrate, also, the homogeneous textured samples simulated under the model (1) with the learnt interaction structures and potentials. Controllable simulated annealing (CSA) technique introduced in $[6,7]$ was used for texture generation. The simulation starts from an IRF sample. The chi-square distances between the normalized GLDHs of the training and simulated samples are reduced from the starting values of $601,000 \ldots 1,329,000$ at the first step of CSA $(t=0)$ to $1425 \ldots 6125$ at the last one $(t=200)$ for the samples in Figure 1 and from $965,000 \ldots 2,712,000$ to $2300 \ldots 7500$ for the samples in Figure 2. As a result, the interaction maps and learnt structures computed for the simulated samples closely match the initial ones for the training samples both in the geometric forms and numbers of the chosen clique families. Therefore, they all represent the same texture type to within the chosen Gibbs model.

It is easily seen that the simulated samples, in spite of their limited visual similarity to the natural ones, reflect basic spatial structure of the training patterns. Notice that the model of Eq. (1) takes account only of the grey level differencr, . Recause the training GLDHs possess an approximate mirror symmetry and hence are almost invariant to inversion of the image grey ranges, the simulated images demonstrate continuous transitions between the initial (training) and inverted image representations. To avoid such changes, a more general image model exploiting pairwise grey level co-occurrences has to be involved. But, because visually the generated samples differ notably from the natural training ones both these textures D3 and D14 are unlikely to be considered as the purely stochastic ones.

Figures 3 and 4 present 36 samples of other natural textures from [2], used as the training ones, and the corresponding simulated samples obtained by the CSA. These experiments show that some these textures, say, D4, D5, D9, D29, D50, D57, D68, D69, D76, D77, D79, D80, D92, D93, really belong to the class of stochastic textures. In other words, the natural and simulated patterns possess both the good visual resemblance and the high proximity between their GLDHs chosen for the Gibbs model of Eq. (1). But generally, such a proximity does not assure the visual similarity which either is limited (as for the textures D24, D65, D74, d82, D83, D84, D105) or does not exist at all. In the latter case the texture is built from some regular texels containing more than a single pixel (D6, D11, D17, D20, D34, D55, D82, D85, D101) or the training sample has substantial local or global inhomogeneities (D23, D36, D66, D75, D95, D103). Therefore, one should discriminate between the homogeneous stochastic textures and all the other types only by such simulation experiments.

Of course, the model-based interaction maps allow to check if there exist most characteristic interactions to represent the given texture type only if $(i)$ the texture is spatially homogeneous, or translation invariant in terms of the local conditional probabilities of the signals, and (ii) if the sample size is sufficiently large to get consistent estimates of them. The interaction map cannot reveal structural features perceived easily by human vision if the local signal configurations are not sufficiently homogeneous over the image. In such a case, 


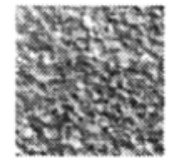

T-D4

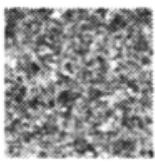

T-D9

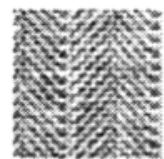

T-D17

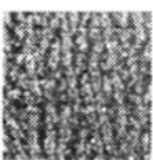

T-D24

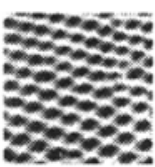

T-D36

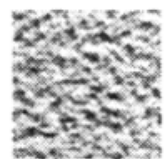

T-D57

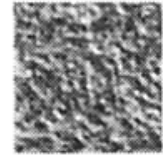

G-D4

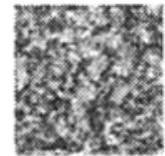

G-D9

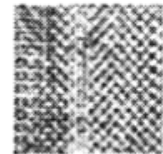

G-D17

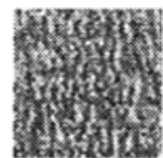

G-D24

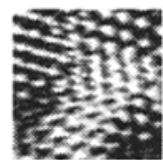

G-D36

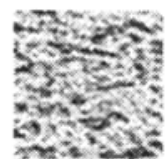

G-D57

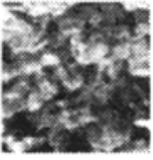

T-D5

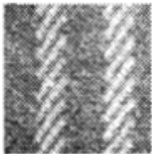

T-D11

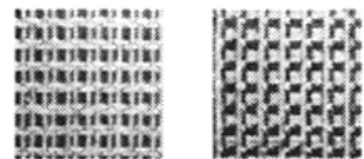

T-D20

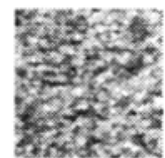

T-D29

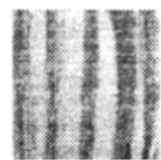

T-D50

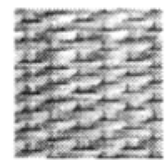

T-D65

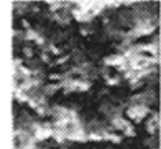

G-D5

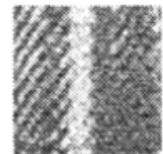

G-D11

G-D20

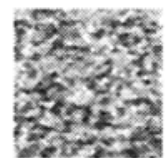

G-D29

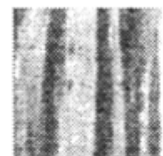

G-D50

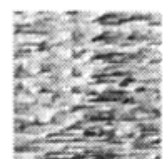

G-D65

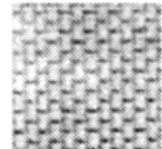

T-D6

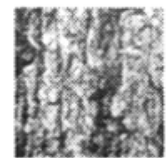

T-D12

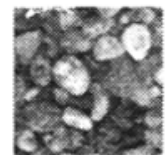

T-D23

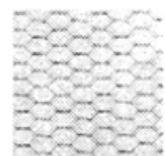

T-D34

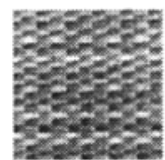

T-D55

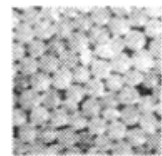

T-D66

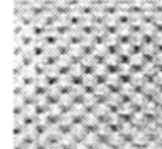

G-D6

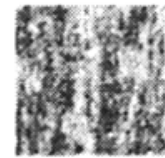

G-D12

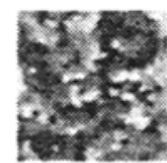

G-D23

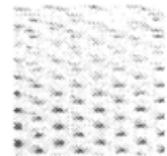

G-D34

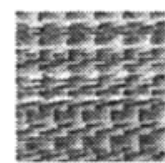

G-D55

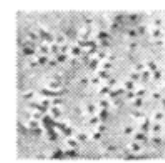

G-D66

Fig. 3. Training (T) and simulated (G) $128 \times 128$ samples of different natural textures.

due to averaging of different non-homogeneous interactions in the GLDHs, the resulting interaction maps and learnt interaction structures do not reflect essential local features of the interactions. Also, a proper choice of the thresholds to reveal the characteristic interaction structures of different textures is not obvious and needs further theoretical investigation.

In spite of these drawbacks, the proposed technique for recovering the interaction structure holds much promise in texture simulating and retrieving $[6,7,9]$. On a basis of this technique, the feature-based interaction maps, derived from 


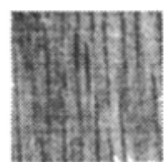

T-D68

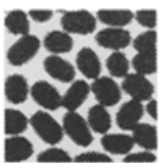

T-D75

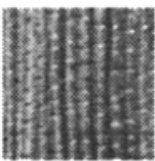

T-D79

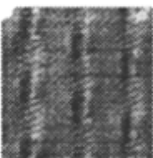

T-D83

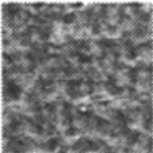

T-D92

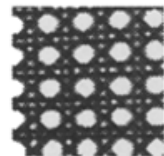

T-D101

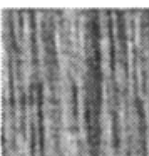

G-D68

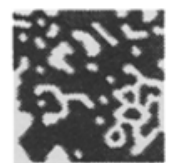

G-D75

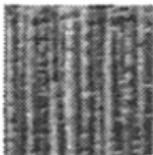

G-D79

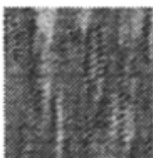

G-D83

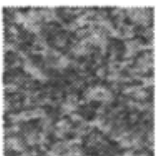

G-D92

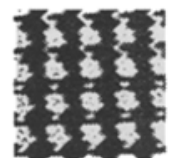

G-D101

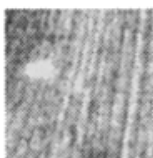

T-D69

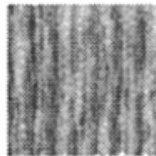

T-D76

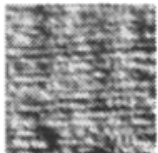

T-D80

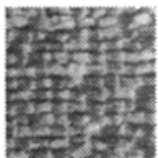

T-D84

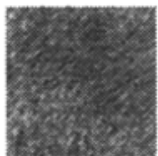

T-D93

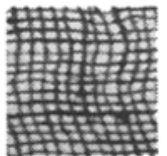

T-D103

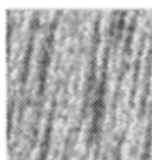

G-D69

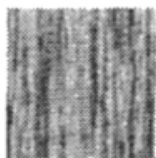

G-D76

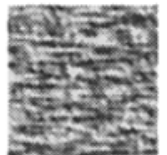

G-D80

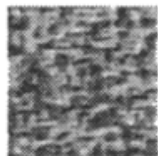

G-D84

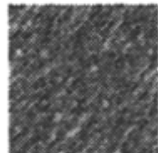

G-D93

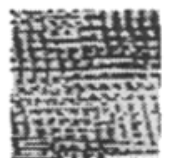

G-D103

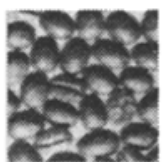

T-D74

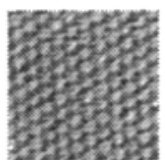

T-D77

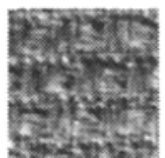

T-D82

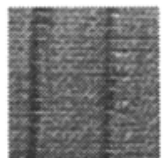

T-D85

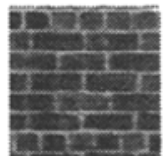

T-D95

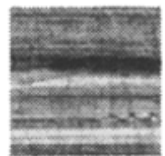

T-D105

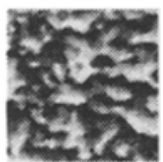

G-D74

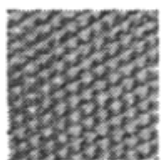

G-D77

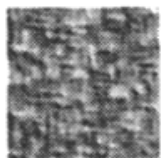

G-D82

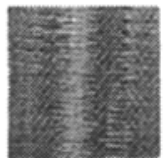

G-D85

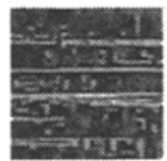

G-D95

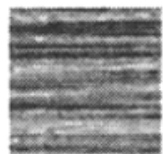

G-D105

Fig. 4. Training (T) and simulated (G) $128 \times 128$ samples of different natural textures.

the extended GLDHs, are introduced recently [5] to analyse such features of the image textures as symmetry, anisotropy, and regularity. In particular, if there exist internal symmetries in the texture patterns then the chosen structures or, at least, the initial interaction map reveals them. But, a quantitative description of such the features needs more theoretical and experimental efforts in spite of a principal possibility to link these structural features with the model-based interaction maps.

The above results allow to conclude that the model-based interaction maps 
possess good potential in texture modelling. They provide the means for recovering a characteristic image structure in terms of pairwise pixel interactions and for measuring quantitatively the similarity between the image patches which are sufficiently large for consistent estimation of marginal probabilities by the GLDHs. Of course, there are many open problems to be solved in future, in particular, how to search for the minimum number of characteristic neighbours that represent a structure of a given texture, how to measure similarity under more diverse than simple translations geometric transformations of the image patches, etc.

\section{References}

1. Besag, J. E.: Spatial interaction and the statistical analysis of lattice systems. J. Royal Statistical Soc. B36 (1974) 192-236

2. Brodatz, P.: Textures: A Photographic Album for Artists an Designers. New York: Dover Publications (1966)

3. Chellappa, R., Jain, A. (Eds): Markov Random Fields: Theory and Application. Academic Press (1993)

4. Chetverikov, D.: On some basic concepts of texture analysis. Proc. 2nd Int. Conf. on Computer Analysis of Images and Patterns. Sept. 2-4, 1987, Wismar, GDR. Berlin: Akademie-Verlag (1987) 196-201

5. Chetverikov, D., Haralick, R. M.: Texture anisotropy, symmetry, regularity: recovering structure and orientation from interaction maps. Proc. 6th British Machine Vision Conf. September 11-14, 1995, Birmingham. Sheffield: Univ. of Sheffield (1995) 57-66.

6. Gimel'farb, G. L.: Texture modeling by multiple pairwise pixel interactions. IEEE Trans. Pattern Anal. Machine Intell. 18:11 (1996) 1110-1114

7. Gimel'farb, G. L.: Non-Markov Gibbs texture model with multiple pairwise pixel interactions. Proc. 13th IAPR Int. Conf. Pattern Recognition, vol. II, Aug.25-29, 1996, Vienna, Austria. TUWien (1996) 591-595

8. Gimel'farb, G. L.: Analytic approximation of Gibbs potentials to model stochastic textures. Proc. First Joint Australia \& New Zealand Biennial Conf. on Digital Image \& Vision Computing: Techniques and Applications (DICTA'97 \& IVCNZ'97), Albany, Auckland, New Zealand, 10-12 Dec. 1997. Massey Univ.: Palmerston North, 1997, 153-158.

9. Gimel'farb G. L., Jain, A. K.: On retrieving textured images from an image data base. Pattern Recognition 29:9 (1996) 1461-1483

10. Haralick, R. M., Shapiro,L. G.: Computer and Robot Vision. Vol. 1. Reading: Addison-Wesley Publ. Co. (1992) 453-507

11. Julesz, B.: Textons, the elements of texture perception, and their interactions. Nature 290 (1981) 91-97

12. Zalesny, A. V.: Homogeneity \& texture. General approach. Proc. 12th IAPR Int. Conf. on Pattern Recognition. October 9-13, 1994, Jerusalem, Israel. Vol. 1. Los Alamitos: IEEE Comp. Soc. Press (1994) 592-594. 